\title{
Alpha Head on Collision with a Fixed Gold Nucleus, Taking into Account the Relativistic Rest Mass Variation as Implied by Mass-Energy Equivalence
}

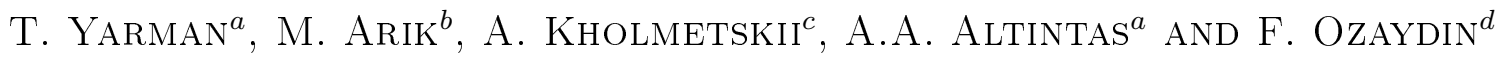 \\ ${ }^{a}$ Okan University, Tuzla, Istanbul, Turkey \\ ${ }^{b}$ Bogazici University, Bebek, Istanbul, Turkey \\ ${ }^{c}$ Belarus State University, Minsk, Belarus \\ ${ }^{d}$ Isik University, Sile, Istanbul, Turkey
}

\begin{abstract}
We reformulate the Rutherford scattering of alpha particle for a head on collision, taking into account the rest mass variation of the particle, as implied by the energy conservation law. Our relativistic reformulation (which includes the energy conservation) constitutes a new example for the breakdown of the Lorentz invariance. Briefly speaking, even at rest or during the whole scattering process, the distance between the alpha particle and the gold nucleus is not invariant but depends on the frame of the observer attached to either object. According to our relativistic reformulation, we also provide a new set of Lorentz transformations.
\end{abstract}

DOI: $10.12693 /$ APhysPolA.125.618

PACS: 21.60.-n, 24.10.Jv

\section{Introduction}

Suppose we have an alpha particle of charge ze (where $z$ is 2 , and $e$ is the fundamental charge), and of rest mass $m_{0 \infty}$, at infinity. It moves with an instantaneous velocity $v_{0}$ at a distance $r_{0}$ from the gold nucleus of charge $Z e$, at the given time, as measured by an observer attached to the alpha particle. As a first approach, we suppose that the alpha particle heads right on $Z e$, just like one could have observed, as in the Rutherford's nucleus discovery experiment [1]. Below, we will reformulate the head on collision, taking into account the rest mass variation of the alpha, throughout, as implied by the mass and energy equivalence of the special theory of relativity (STR). Thus, we first express the rest energy of alpha at the given distance from the gold nucleus, and the total relativistic energy of it, given that it is in motion, except at the moment it is stopped by the gold nucleus. Then we deal with the quest of how the internal dynamics of alpha and its size is affected through the process.

\section{Rest and total relativistic energies of alpha}

According to our approach the rest energy of the alpha particle is

$$
m_{0}\left(r_{0}\right) c^{2}=m_{0 \infty} c^{2}\left(1+\frac{Z z e^{2}}{r_{0} m_{0 \infty} c^{2}}\right) .
$$

In the above formula the observer is attached to alpha particle and measures the distance between alpha and gold nucleus as $r_{0}$. In our approach for an observer which is located on the gold nucleus the distance between gold nucleus and alpha particle is measured as $r$ which is different from $r_{0}$.

Now the total relativistic energy of alpha particle is written by applying Lorentz mass increase to Eq. (1)

$$
m_{\gamma} c^{2}=\frac{m_{0 \infty} c^{2}\left(1+\frac{Z z e^{2}}{r_{0} m_{0 \infty} c^{2}}\right)}{\sqrt{1-\frac{v_{0}^{2}}{c^{2}}}}=\frac{m_{0 \infty} c^{2}}{\sqrt{1-\frac{v_{00}^{2}}{c^{2}}}}=\text { const, }
$$

where $v_{00}$ is the initial velocity of the alpha particle, far away from the gold nucleus.

Since the system is closed, the total energy is constant; this means that at the distance $R_{0}$, too (the alpha particle is stopped), Eq. (2) remains constant

$$
m_{\gamma} c^{2}=m_{0 \infty} c^{2}\left(1+\frac{Z z e^{2}}{R_{0} m_{0 \infty} c^{2}}\right)=\text { const. }
$$

Let us for simplicity pose

$$
\chi\left(r_{0}\right)=\left(1+\frac{Z z e^{2}}{r_{0} m_{0 \infty} c^{2}}\right), \quad \text { and } \quad \gamma\left(v_{0}\right)=\sqrt{1-\frac{v_{0}^{2}}{c^{2}}} .
$$

In the head on collision the alpha particle is stopped by gold nucleus at $R_{0}$ and then fired back, after which Eq. (3) still remains valid. Via Eq. (3) one can write the equation of motion.

\subsection{Equation of motion}

Thus, by differentiating Eq. (3) one can get

$$
\frac{Z z e^{2}}{r_{0}^{2}} \sqrt{1-\frac{v_{0}^{2}}{c^{2}}}=m_{0 \infty}\left(1+\frac{Z z e^{2}}{r m_{0 \infty} c^{2}}\right) \frac{\frac{\mathrm{d} v_{0}}{\mathrm{~d} t_{0}}}{\sqrt{1-\frac{v_{0}^{2}}{c^{2}}}} .
$$

Using Eq. (2), we have

$$
\frac{Z z e^{2}}{r_{0}^{2}} \sqrt{1-\frac{v_{0}^{2}}{c^{2}}}=m_{\gamma} \frac{\mathrm{d} v_{0}}{\mathrm{~d} t_{0}}
$$

In our approach, unlike the classical approach, the measured distances depend on observers $[2,3]$ attached to the gold nucleus, or the alpha particle. In such a framework, for the outside observer the measured distance is 
written as

$$
r=\frac{r_{0}}{1+\frac{Z z e^{2}}{r_{0} m_{0 \infty} c^{2}}} .
$$

The velocities in both frames are the same, thus periods of time change as much as the distances are altered [4]. With the help of Eq. (7), Eq. (6) becomes

$$
\frac{Z z e^{2}}{r^{2}\left(1+\frac{Z z e^{2}}{r_{0} m_{0 \infty} c^{2}}\right)} \sqrt{1-\frac{v_{0}^{2}}{c^{2}}}=m_{\gamma} \frac{\mathrm{d} v}{\mathrm{~d} t\left(1+\frac{Z z e^{2}}{r_{0} m_{0 \infty} c^{2}}\right)} \text {. }
$$

Using Eq. (2), the equation of motion in the outside observer's reference frame will be

$$
\frac{Z z e^{2}}{r^{2}} \frac{1}{\left(1+\frac{Z z e^{2}}{R_{0} m_{0 \infty} c^{2}}\right)}=m_{\gamma} \frac{\mathrm{d} v}{\mathrm{~d} t} .
$$

It is easy to see that equation of motions depends on the frame of observer. Equation (6) is the equation of motion written in the frame of alpha particle and Eq. (9) is equation of motion written in the frame of outside observer.

\subsection{Differences in Lagrangians}

Since the equation of motion varies according to observer's frame, a natural quest arises on, what happens to Lagrangian? The definition of Lagrangian is $L=K-U$, here $K$ is kinetic energy of alpha particle and $U$ is the electrostatic potential energy. Since the total energy $E=K+U$, the Lagrangian turns into $L=E-2 U$. Since the $U$ term depends on the observer, the difference in Lagrangians is just the difference in $U$ :

$$
\Delta U=\frac{Z z e^{2}}{r}\left(\sqrt{1-\frac{v_{0}^{2}}{c^{2}}}-\sqrt{1-\frac{v_{00}^{2}}{c^{2}}}\right) .
$$

So it is zero at infinity, but not so, at closer ranges. This answers a possible question with regards to how the classical Lagrangian, and accordingly the action principle are to be modified to cope with our approach.

In any case, we wanted to show that the classically believed Lorentz symmetry, is broken in our approach, even at rest; indeed, when the alpha is stopped by the gold nucleus, the distance measured by the observer attached to the alpha, from this to the gold nucleus, is different from the same distance measured by the observer attached to the gold nucleus.

Therefore, the equations of motion written by the two observers with regards to the same head on collision, will turn out to be different. The corresponding Lagrangians and action principles, too, then, must be different.

\subsection{Modified Lorentz transformations}

In this section we propose a new set of the Lorentz transformations which also depends on the interaction. This means that the Lorentz transformations should also have interaction terms. By using parameters which were defined in Eq. (4) the differential form of new Lorentz transformations is

$$
\begin{aligned}
& \mathrm{d} x^{\prime}=\gamma \chi(\mathrm{d} x-v \mathrm{~d} t), \\
& \mathrm{d} y^{\prime}=\mathrm{d} y=0, \\
& \mathrm{~d} z^{\prime}=\mathrm{d} z=0, \\
& \mathrm{~d} t^{\prime}=\gamma \chi\left(\mathrm{d} t-v \frac{v \mathrm{~d} x}{c^{2}}\right) .
\end{aligned}
$$

$$
\begin{aligned}
& x^{\prime}=\gamma \chi\left(x-\int_{0}^{t} v \mathrm{~d} t\right) R \chi_{0}, \\
& y^{\prime}=y \\
& z^{\prime}=z \\
& t^{\prime}=\gamma \chi\left(t-\int_{0}^{t} \frac{v \mathrm{~d} x}{c^{2}}\right) .
\end{aligned}
$$

The inverse transformations can also be written easily.

\section{Conclusion}

We have thus reformulated the Rutherford scattering of alpha particles, as a first approach, for a head on collision, yet taking into account the rest mass variation of the alpha throughout, as implied by the mass and energy equivalence of the special theory of relativity. Our approach predicts the same impact parameter as the classical one, though when seen from the alpha particle. Yet our impact parameter when seen by the distant observer (or the same, from the gold nucleus, unaltered by the repelled alpha), differs from the classical one.

The relative difference between $R$ and $R_{0}$ is

$$
\begin{aligned}
& \frac{R-R_{0}}{R_{0}}=-\frac{Z z e^{2}}{R_{0} m_{0 \infty} c^{2}}=-\frac{1}{2} m_{0 \infty} v_{0}^{2}=-\frac{1}{2}\left(\frac{v_{0}}{c}\right)^{2} \\
& \quad \approx 2 \times 10^{-3} .
\end{aligned}
$$

The original velocity $v_{0}$ of alpha in the Rutherford experiment is about $2 \times 10^{7} \mathrm{~m} / \mathrm{s}$.

The framework we draw, anyway constitutes an example for the breakdown of the "Lorentz invariance". Finally we have provided a modified set of Lorentz transformations, involving not only the motion but also the interaction between particles of concern.

\section{Acknowledgments}

This work was supported by the Scientific Research Projects Coordination Unit of Istanbul University, project number: BAP-5623.

\section{References}

[1] E. Rutherford, Philos. Mag. 6, 21 (1911).

[2] F. Ozaydin, A.A. Altintas, L.A. Susam, M. Arik, T. Yarman, AIP Conf. Proc. 1476, 361 (2012).

[3] T. Yarman, M. Arik, A.A. Altintas, F. Ozaydin, in: Physics of Reality: Space, Time, Matter, Cosmos - Proceedings of the 8th Symposium Honoring Mathematical Physicist Jean-Pierre Vigier, Ed. R. Amoroso, World Scientific, Singapore 2013.

[4] T. Yarman, Found. Phys. Lett. 19, 675 (2006).

From above transformations one can get 